\title{
Fitossociologia e estrutura diamétrica de um fragmento de Cerrado sensu stricto, Formoso do Araguaia, Tocantins
}

\section{Phytosociology and diametric structure of a fragment of Cerrado sensu stricto, Formoso do Araguaia, Tocantins, Brazil}

\author{
Rafaella da Costa Gama*1, Thiêssa Tamilla de Carvalho Santana², Tayanne Graciette Nascimento Silva², Valdir Carlos Lima \\ de Andrade ${ }^{4}$, Priscila Bezerra de Souza ${ }^{5}$
}

\begin{abstract}
Resumo: O Cerrado Brasileiro é composto por diferentes fitofisionomias, onde observa-se vegetação campestre, savânica e florestal. Assim, objetivou-se realizar um levantamento fitossociológico para avaliar a estrutura diamétrica de um fragmento de Cerrado sensu stricto, Formoso do Araguaia - TO. Foram instaladas, aleatoriamente, seis parcelas retangulares de $500 \mathrm{~m}^{2}$ cada $(10 \times 50 \mathrm{~m})$, totalizando 0,3 ha de área amostral. Nas parcelas amostrou-se todos os indivíduos arbustivos-arbóreos vivos e mortos em pé com Circunferência à Altura do Peito (CAP) $\geq 10 \mathrm{~cm}$. Foram 508 indivíduos arbustivos-arbóreos amostrados, sendo 480 vivos e 28 mortos em pé, distribuídos em 49 espécies e 27 famílias. As principais famílias foram Vochysiaceae, Fabaceae, Malpighiaceae, Sapotaceae e Melastomataceae. A Qualea parviflora Mart., Qualea grandiflora Mart., Pouteria ramiflora (Mart.) Radlk., Byrsonima coccolobifolia Kunth e Sclerolobium paniculatum Vogel apresentaram os maiores Valores de Importância. O diâmetro médio dos indivíduos foi de $9,38 \mathrm{~cm}$, a área basal de $4,71 \mathrm{~m}^{2} \mathrm{ha}^{-1}$ e densidade de $1.693,33$ indivíduos ha ${ }^{-1}$. O índice de diversidade de Shannon Wiener (H') foi de 3,21 e a equabilidade de Pielou (J) de 0,83. Com base nos resultados obtidos observou-se que o fragmento tem elevada diversidade com baixa dominância ecológica e o padrão de distribuição diamétrica "J" invertido foi observado na comunidade evidenciando-se populações estáveis e auto regenerativas.
\end{abstract}

Palavras-chave: Levantamento fitossociológico; estrutura horizontal; savana.

\begin{abstract}
The Brazilian Cerrado is composed of different phytophysiognomies, where one observes country, savanna and forest vegetation. Thus, a phytosociological survey was carried out to evaluate the diametric structure of a Cerrado fragment sensu stricto, Formoso do Araguaia - TO. Six rectangular plots of $500 \mathrm{~m}^{2}$ each $(10 \times 50 \mathrm{~m})$ were installed randomly, totaling 0.3 ha of sample area. In the plots we sampled all live and dead standing shrub trees with Circumference at Breast Height $(\mathrm{CBH}) \geq$ $10 \mathrm{~cm}$. There were 508 shrub-tree individuals sampled, being 480 live and 28 standing dead, distributed in 49 species and 27 families. The main families were Vochysiaceae, Fabaceae, Malpighiaceae, Sapotaceae and Melastomataceae. The Qualea parviflora Mart., Qualea grandiflora Mart., Pouteria ramiflora (Mart.) Radlk., Byrsonima coccolobifolia Kunth and Sclerolobium paniculatum Vogel presented the highest Values of Importance. The mean diameter of the individuals was 9.38 $\mathrm{cm}$, the basal area was $4.71 \mathrm{~m}^{2} \mathrm{ha}^{-1}$ and density was $1,693.33$ individuals $\mathrm{ha}^{-1}$. The diversity index of Shannon Wiener $\left(\mathrm{H}^{\prime}\right)$ was 3.21 and the Pielou (J) equability was 0.83 . Based on the results obtained it was observed that the fragment has high diversity with low ecological dominance and the inverted " $\mathrm{J}$ " diametric distribution pattern was observed in the community evidencing stable and auto regenerative populations.
\end{abstract}

Keywords: Phytosociologic; horizontal structure; savanna.

\footnotetext{
*Autor para correspondência

Recebido para publicação em 26/03/2018; aprovado em 26/09/2018

${ }^{1}$ Eng, Florestal, Mestranda em Ciências Florestais e Ambientais, Universidade Federal do Tocantins, Gurupi, (63) 9 8475-2742, rafaellacg@yahoo.com.br

${ }^{2}$ Eng. Florestal, Mestranda em Ciências Florestais e Ambientais, Universidade Federal do Tocantins, Gurupi, (63) 98125-6061, tamillathiessa@ gmail.com

${ }^{3}$ Eng. Ambiental, Mestranda em Ciências Florestais e Ambientais, Universidade Federal do Tocantins, Gurupi, (99) 98429-8800, thy.graciette@ gmail.com

${ }^{4}$ Eng. Florestal, Dr. Professor do Curso de Engenharia Florestal e do Programa de Pós-Graduação em Ciências Florestais e Ambientais, Universidade Federal do Tocantins, Gurupi, (63) 3311-1714, vclandrade@uft.edu.br

${ }^{5}$ Bióloga, Dra. Professora do Curso de Engenharia Florestal e do Programa de Pós-Graduação em Ciências Florestais e Ambientais, Universidade Federal do Tocantins, Gurupi, (63) 3311-1726, priscilauft@uft.edu.br
} 


\section{INTRODUÇÃO}

O bioma Cerrado é o segundo maior em extensão, superado apenas pela Amazônia, ocupando aproximadamente $21 \%$ do território brasileiro, constituído por um mosaico de formações vegetais que variam de campos abertos a formações florestais densas, contando com mais de 7.000 espécies e elevado grau de endemismo (KLINK: MACHADO, 2005; RIBEIRO; WALTER, 2008). Atualmente, trata-se do bioma mais descaracterizado e ocupado, com a substituição de várias de suas fisionomias por pastagem e produção agrícola, em decorrência, principalmente, da política governamental de incentivo agrícola no Cerrado a partir da década de 1970 (MMA, 2015).

Em áreas de Cerrado é possível encontrar diferentes formas de vegetação, que variam quanto a sua fisionomia, estrutura e florística, observa-se uma vegetação campestre, como o Campo Limpo, savânica como o Campo Sujo, Campo Cerrado e Cerrado sensu stricto e vegetação florestal como o Cerradão (COUTINHO, 2006). Entre as fisionomias do Cerrado as mais visadas são o Cerradão e o Cerrado sensu stricto para instalação de áreas de pecuária e agricultura (CARVALHO et al., 2016).

A análise da fitossociologia das comunidades vegetais surgiu com a necessidade de conhecer os biomas em que estão inseridas e retratar a composição, estrutura e distribuição das espécies (FELFILI et al., 2002). De modo que os estudos fitossociológicos podem auxiliar na conservação e manejo de áreas remanescentes, para recuperação de áreas degradadas, licenciamento ambiental, identificação de espécies ameaçadas e outros (BRITO et al., 2007).

Florestas inequiâneas, em especial as tropicais, que não sofreram muitas perturbações apresentam distribuição diamétrica dos indivíduos na forma exponencial negativa, ou seja, se assemelha a forma de "J" invertido, sendo que a maior frequência dos indivíduos está nas menores classes de diâmetro (ALVES JÚNIOR et al., 2007). De acordo com Meyer et al. (1961) a estrutura diamétrica revela a história da comunidade vegetal, podendo indicar o equilíbrio ou desequilíbrio. Além disso, conforme Paula et al. (2004), Siminski et al. (2004) e Machado et al. (2010), a distribuição diamétrica é base para explicar a estrutura de uma determinada área florestal e entender a sua dinâmica sucessional. Também, é importante para se realizar inferências sobre seu desenvolvimento e sua sustentabilidade.

Diante desse contexto objetivou-se realizar um levantamento fitossociológico para avaliar a estrutura diamétrica de um fragmento de Cerrado sensu stricto, Formoso do Araguaia - TO.

\section{MATERIAL E MÉTODOS}

O presente trabalho foi realizado em um fragmento de Cerrado sensu stricto na área de Reserva Legal da Fazenda Bom Sossego no Município de Formoso do Araguaia - TO, sob as coordenadas $11^{\circ} 53^{\prime} 25.2^{\prime \prime}$ latitude sul e 49²5'32.6" longitude oeste.

O clima predominante da região é $\mathrm{C} 2 \mathrm{wA}^{\prime} \mathrm{a}$ ', úmido subúmido com duas estações bem definidas, uma seca de maio a setembro e uma chuvosa de outubro a abril, com moderada deficiência hídrica no inverno, precipitação média anual em torno de 1.600 a $1.700 \mathrm{~mm}$, com temperatura média anual entre 25 e $26^{\circ} \mathrm{C}$ e $240 \mathrm{~m}$ de altitude (SEPLAN, 2012).

Foram alocadas aleatoriamente, em uma área com 20,2 ha de Cerrado sensu stricto, seis parcelas retangulares de 500 $\mathrm{m}^{2}$ cada $(10 \times 50 \mathrm{~m})$, totalizando 0,3 ha de área amostral (SANTOS et al., 2010; LIMA; LEÃO, 2013). Nas parcelas foram amostrados todos os indivíduos arbustivos-arbóreos vivos e mortos em pé com Circunferência à Altura do Peito $(\mathrm{CAP}) \geq 10 \mathrm{~cm}$.

A identificação das espécies foi realizada em campo com auxílio de especialistas da área e literatura pertinente (MEDEIROS, 2011; LIMA et al., 2013). A lista florística foi organizada com base nas espécies reconhecidas pelo Angiosperm Phylogeny Group III (APG III, 2009).

Os parâmetros fitossociológicos analisados, obtidos a partir do emprego do Softwar Fitopac versão 2.1.2 (SHEPHERD, 2010), foram: densidade relativa (DR), densidade absoluta (DA), dominância relativa (DoR), dominância absoluta (DoA), frequência relativa (FR), frequência absoluta (FA) e índice de valor de importância (IVI), calculados pelas formas tradicionais, além desses parâmetros calculou-se o índice de diversidade de Shannon (H') e Pielou (J).

Os diâmetros obtidos após a transformação da circunferência $(\mathrm{DAP}=\mathrm{CAP} / \mathrm{pi})$, foram distribuídos em classes diamétricas empregando-se a regra de Sturges utilizando a Equação (1) (MONTEIRO FILHO; ROCHA, 2016; CERQUEIRA et al., 2017).

$$
\mathrm{n}_{\mathrm{c}}=1+3,3 \log (\mathrm{n})
$$

Em que: $n_{c}$ é o número de classes e $n$ é o número de indivíduos.

O número de classes de diâmetro calculado pela regra de Sturges, com n igual a 508 (número de indivíduos amostrados) foi de 10,02. O maior valor observado foi de $48,80 \mathrm{~cm}$ e o menor de $3,02 \mathrm{~cm}$. A amplitude observada foi de 45,68 cm. O resultado da divisão desse valor por 10,02 é $4,58 \mathrm{~cm}$. Logo, foram obtidas 10 classes com intervalo de $4,58 \mathrm{~cm}$.

\section{RESULTADOS E DISCUSSÃO}

Foram amostrados em 0,3 ha 508 indivíduos arbustivosarbóreos, sendo identificados 480 indivíduos vivos e 28 indivíduos mortos em pé, distribuídos em 49 espécies e 27 famílias, com diâmetro médio de $9,38 \mathrm{~cm}$, correspondendo a uma área basal de 4,71 $\mathrm{m}^{2} \mathrm{ha}^{-1}$ e densidade de 1.693,3 ind. ha 1 . Dessa forma, cabe ressaltar que a densidade encontrada neste estudo foi superior a comumente observada em Cerrado sensu stricto, de acordo com Felfili (2008) a densidade em áreas de Cerrado sensu stricto estão entre os intervalos de 400 a 1.000 ind. ha $^{-1}$ e para área basal são representativos na vegetação lenhosa um valor de 3 a $8 \mathrm{~m}^{2} \mathrm{ha}^{-1}$.

$\mathrm{O}$ valor do índice de diversidade de Shannon Wiener (H') foi de 3,21 e a equabilidade de Pielou de 0,83, valores que se divergem ligeiramente aos encontrados por outros autores em Cerrado sensu stricto (MEDEIROS; WALTER, 2012; FERREIRA et al., 2015; SILVA; SOUZA, 2016). Com base no elevado valor do índice de Shannon é possível afirmar uma alta diversidade na área pesquisada. Além disso, pode-se inferir que a baixa dominância ecológica é evidenciada pelo elevado valor de equabilidade (GIÁCOMO et al., 2013). 
Quanto à mortalidade de árvores que, de acordo com Martins (1991), ocorre devido a ventos, tempestades, doenças, perturbações antrópicas ou senescência. Foram encontrados 28 indivíduos mortos em pé que totalizaram $5,51 \%$ de todos os indivíduos amostrados, valor que não destoa da literatura, visto que, Silva e Souza (2016) encontraram 3,10\% de árvores mortas em fragmento de Cerrado sensu stricto em Gurupi - TO. Silva Neto et al. (2016b), também em Gurupi - TO, observaram 7,26\% de mortos em pé em Cerrado sensu stricto. Com base na frequência absoluta, pode-se observar que ocorreram árvores mortas em 100\% das parcelas, indicando não haver uma perturbação localizada na área estudada.

Excluindo o grupo das árvores mortas, as espécies Qualea parviflora Mart., Qualea grandiflora Mart., Pouteria ramiflora (Mart.) Radlk., Byrsonima coccolobifolia Kunth, Curatella americana L., Psidium cattleianum Sabine, Ouratea hexasperma (A. St. Hil.) Baill., Miconia albicans (Sw.) Triana, Sclerolobium paniculatum Vogel e Caryocar brasiliense Cambess. apresentaram os maiores valores de densidade absoluta, representando $20,83 \%$ de todas as espécies amostradas, além de reunirem $67,29 \%$ da densidade absoluta total (Tabela 1).

Tabela 1. Parâmetros fitossociológicos das espécies amostradas em 0,3 ha de Cerrado sensu stricto em ordem decrescente de IVI = valor de importância $(\%) ; \mathrm{DA}=$ densidade absoluta (número de indivíduos/ha); $\mathrm{DR}=$ densidade relativa $(\%)$;oA = dominância absoluta $\left(\mathrm{m}^{2} / \mathrm{ha}\right) ; \mathrm{DoR}=$ dominância relativa $(\%) ; \mathrm{FA}=$ frequência absoluta $(\%)$ e FR= frequência relativa $(\%)$

\begin{tabular}{|c|c|c|c|c|c|c|c|}
\hline Espécie & DA & DR & DoA & DoR & FA & FR & IVI \\
\hline Qualea parviflora Mart. & 203,3 & 12,01 & 3,48 & 22,19 & 100,00 & 4,38 & 12,86 \\
\hline Qualea grandiflora Mart. & 216,7 & 12,80 & 1,18 & 7,52 & 100,00 & 4,38 & 8,23 \\
\hline Pouteria ramiflora (Mart.) Radlk. & 163,3 & 9,65 & 0,86 & 5,50 & 100,00 & 4,38 & 6,51 \\
\hline Grupo das Mortas & 93,3 & 5,51 & 0,97 & 6,19 & 100,00 & 4,38 & 5,36 \\
\hline Byrsonima coccolobifolia Kunth & 90,0 & 5,31 & 1,10 & 7,01 & 83,33 & 3,65 & 5,32 \\
\hline Sclerolobium paniculatum Vogel & 56,7 & 3,35 & 0,95 & 6,07 & 100,00 & 4,38 & 4,60 \\
\hline Curatella americana $\mathrm{L}$. & 83,3 & 4,92 & 0,70 & 4,49 & 100,00 & 4,38 & 4,60 \\
\hline Psidium cattleianum Sabine & 80,0 & 4,72 & 0,49 & 3,15 & 100,00 & 4,38 & 4,09 \\
\hline Ouratea hexasperma (A. St. Hil.) Baill. & 76,7 & 4,53 & 0,26 & 1,68 & 100,00 & 4,38 & 3,53 \\
\hline Caryocar brasiliense Cambess. & 43,3 & 2,56 & 0,53 & 3,35 & 100,00 & 4,38 & 3,43 \\
\hline Vatairea macrocarpa (Benth.) Ducke & 30,0 & 1,77 & 0,43 & 2,71 & 100,00 & 4,38 & 2,96 \\
\hline Miconia albicans (Sw.) Triana & 63,3 & 3,74 & 0,26 & 1,65 & 66,67 & 2,92 & 2,77 \\
\hline Magonia pubescens A.St.-Hil. & 40,0 & 2,36 & 0,43 & 2,73 & 50,00 & 2,19 & 2,43 \\
\hline Dimorphandra mollis Benth. & 33,3 & 1,97 & 0,46 & 2,94 & 50,00 & 2,19 & 2,37 \\
\hline Caesalpinia pyramidalis Tul. & 40,0 & 2,36 & 0,23 & 1,49 & 50,00 & 2,19 & 2,01 \\
\hline Protium heptaphyllum (Aubl.) Marchand & 40,0 & 2,36 & 0,33 & 2,13 & 33,33 & 1,46 & 1,99 \\
\hline Aspidosperma tomentosum Mart. & 30,0 & 1,77 & 0,31 & 1,98 & 50,00 & 2,19 & 1,98 \\
\hline Byrsonima intermedia A.Juss. & 30,0 & 1,77 & 0,15 & 0,97 & 66,67 & 2,92 & 1,89 \\
\hline Mouriri apiranga Spruce ex Triana & 16,7 & 0,98 & 0,37 & 2,39 & 33,33 & 1,46 & 1,61 \\
\hline Roupala montana Aubl. & 26,7 & 1,57 & 0,19 & 1,24 & 33,33 & 1,46 & 1,43 \\
\hline Xylopia aromatica (Lam.) Mart. & 23,3 & 1,38 & 0,09 & 0,56 & 50,00 & 2,19 & 1,38 \\
\hline Astronium fraxinifolium Schott ex Spreng & 10,0 & 0,59 & 0,20 & 1,31 & 50,00 & 2,19 & 1,36 \\
\hline Hancornia speciosa Gomes & 16,7 & 0,98 & 0,05 & 0,32 & 50,00 & 2,19 & 1,17 \\
\hline Tabebuia aurea (Silva Manso) Benth \& Hook f. ex S. Moore & 13,3 & 0,79 & 0,07 & 0,45 & 50,00 & 2,19 & 1,14 \\
\hline Annona coriacea Mart. & 13,3 & 0,79 & 0,04 & 0,28 & 50,00 & 2,19 & 1,09 \\
\hline Apeiba tibourbou Aubl. & 13,3 & 0,79 & 0,04 & 0,23 & 50,00 & 2,19 & 1,07 \\
\hline Strychnos pseudoquina A.St.-Hil. & 16,7 & 0,98 & 0,07 & 0,46 & 33,33 & 1,46 & 0,97 \\
\hline Pterodon emarginatus Vogel & 6,7 & 0,39 & 0,13 & 0,84 & 33,33 & 1,46 & 0,90 \\
\hline Guapira graciliflora (Mart. Ex Schmidt) & 6,7 & 0,39 & 0,21 & 1,35 & 16,67 & 0,73 & 0,82 \\
\hline Dipteryx alata Vogel & 10,0 & 0,59 & 0,06 & 0,39 & 33,33 & 1,46 & 0,81 \\
\hline Ipomoea carnea Jacq. & 3,3 & 0,20 & 0,20 & 1,30 & 16,67 & 0,73 & 0,74 \\
\hline Cochlospermum regium (Schrank) Pilg. & 6,7 & 0,39 & 0,17 & 1,06 & 16,67 & 0,73 & 0,73 \\
\hline Hymenaea stilbocarpa Mart. Ex Hayne & 6,7 & 0,39 & 0,15 & 0,99 & 16,67 & 0,73 & 0,70 \\
\hline Byrsonima affinis W.R.Anderson & 10,0 & 0,59 & 0,12 & 0,75 & 16,67 & 0,73 & 0,69 \\
\hline Byrsonima crassifolia (L.) Kunth & 6,7 & 0,39 & 0,03 & 0,20 & 33,33 & 1,46 & 0,69 \\
\hline Copaifera langsdorfii Desf. & 6,7 & 0,39 & 0,03 & 0,19 & 33,33 & 1,46 & 0,68 \\
\hline Mouriri pusa Gard. & 3,3 & 0,20 & 0,09 & 0,60 & 16,67 & 0,73 & 0,51 \\
\hline Garcinia brasiliensis Mart. & 10,0 & 0,59 & 0,03 & 0,17 & 16,67 & 0,73 & 0,50 \\
\hline Antonia ovata Pohl & 10,0 & 0,59 & 0,02 & 0,11 & 16,67 & 0,73 & 0,48 \\
\hline Salacia crassifolia (Mart. ex Schult.) G.Don & 6,7 & 0,39 & 0,02 & 0,11 & 16,67 & 0,73 & 0,41 \\
\hline Bowdichia virgilioides Kunth & 3,3 & 0,20 & 0,05 & 0,31 & 16,67 & 0,73 & 0,41 \\
\hline Brosimum rubescens Taub. & 6,7 & 0,39 & 0,02 & 0,10 & 16,67 & 0,73 & 0,41 \\
\hline Tabebuia ochracea (Cham.) Standl. & 6,7 & 0,39 & 0,01 & 0,06 & 16,67 & 0,73 & 0,40 \\
\hline Himatanthus obovatus (Müll.Arg.) Woodson & 3,3 & 0,20 & 0,04 & 0,24 & 16,67 & 0,73 & 0,39 \\
\hline Genipa americana $\mathrm{L}$. & 3,3 & 0,20 & 0,02 & 0,11 & 16,67 & 0,73 & 0,35 \\
\hline Anacardium occidentale $\mathrm{L}$. & 3,3 & 0,20 & 0,01 & 0,05 & 16,67 & 0,73 & 0,33 \\
\hline Diospyros hispida A.DC. & 3,3 & 0,20 & 0,01 & 0,04 & 16,67 & 0,73 & 0,32 \\
\hline Brosimum gaudichaudii Trécul & 3,3 & 0,20 & 0,00 & 0,03 & 16,67 & 0,73 & 0,32 \\
\hline Eugenia dysenterica Dc. & 3,3 & 0,20 & 0,00 & 0,02 & 16,67 & 0,73 & 0,32 \\
\hline Total & 1693,2 & 100,0 & 15,66 & 100,00 & 2283,37 & 100,00 & 100,00 \\
\hline
\end{tabular}


Quanto a dominância absoluta, os maiores valores foram registrados para as espécies Qualea parviflora Mart. (3,48 $\mathrm{m}^{2} / \mathrm{ha}$ ), Qualea grandiflora Mart. (1,18 $\left.\mathrm{m}^{2} \mathrm{ha}^{-1}\right)$, Byrsonima coccolobifolia Kunth $\left(1,10 \quad \mathrm{~m}^{2} \quad \mathrm{ha}^{-1}\right), \quad$ Sclerolobium paniculatum Vogel $\left(0,95 \mathrm{~m}^{2} \mathrm{ha}^{-1}\right)$, Pouteria ramiflora (Mart.) Radlk. (0,86 $\left.\mathrm{m}^{2} \mathrm{ha}^{-1}\right)$, Curatella americana L. $\left(0,70 \mathrm{~m}^{2} \mathrm{ha}^{-1}\right)$, Caryocar brasiliense Cambess. $\left(0,53 \mathrm{~m}^{2} \mathrm{ha}^{-1}\right)$, Psidium cattleianum Sabine $\left(0,49 \mathrm{~m}^{2} \mathrm{ha}^{-1}\right)$, Dimorphandra mollis Benth. $\left(0,46 \mathrm{~m}^{2} \mathrm{ha}^{-1}\right)$ e Vatairea macrocarpa (Benth.) Ducke $\left(0,43 \mathrm{~m}^{2} \mathrm{ha}^{-1}\right)$.

As espécies melhor distribuídas na área amostrada, ou seja, presentes em $100 \%$ das parcelas alocadas, com exceção da Vatairea macrocarpa (Benth.) Ducke (83,33\% das parcelas), foram: Qualea parviflora Mart., Qualea grandiflora Mart., Pouteria ramiflora (Mart.) Radlk., Byrsonima coccolobifolia Kunth, Sclerolobium paniculatum Vogel, Curatella americana L., Psidium cattleianum Sabine, Ouratea hexasperma (A. St. Hil.) Baill. e Caryocar brasiliense Cambess..

Considerando o IVI, as dez espécies com valores mais elevados, que representaram 20,8\% das espécies amostradas e somando $55,86 \%$ do IVI total (Tabela 1) são: Qualea parviflora Mart., Qualea grandiflora Mart., Pouteria ramiflora (Mart.) Radlk., Byrsonima coccolobifolia Kunth, Sclerolobium paniculatum Vogel, Curatella americana L., Psidium cattleianum Sabine, Ouratea hexasperma (A. St. Hil.) Baill. e Caryocar brasiliense Cambess. e Vatairea macrocarpa (Benth.) Ducke.

As espécies de maior IVI apresentam ampla distribuição nas regiões de cerrados no sudoeste do Tocantins. Dados estes que corroboram com Silva Neto et al. (2016a) e Silva Neto et al. (2016b) onde também encontraram as espécies Qualea parviflora Mart., Qualea grandiflora Mart., Pouteria ramiflora (Mart.) Radlk., Caryocar brasiliense Cambess. e Curatella americana L., com maior IVI.As espécies Qualea grandiflora Mart. e Qualea parviflora Mart. são amplamente distribuídas no Cerrado e destacam-se pela importância florística e fisionômica neste bioma, ocorrendo em mais de $50 \%$ das áreas amostradas (RATTER et al., 2003) assertiva que corrobora com os dados supracitados.

As famílias Vochysiaceae, Fabaceae, Malpighiaceae, Sapotaceae e Melastomataceae apresentaram os melhores resultados quanto aos parâmetros fitossociológicos avaliados (Tabela 2).

Tabela 2. Parâmetros fitossociológicos das famílias em 0,3 ha de Cerrado sensu stricto em ordem decrescente de IVI = valor de importância (\%); $\mathrm{N}^{\mathrm{o}} \mathrm{Sp}=$ número de espécies; \% $\mathrm{Sp} .=$ porcentagem de espécies; DA= densidade absoluta (número de indivíduos/ha); $\mathrm{DR}=$ densidade relativa $(\%) ; \mathrm{DoA}=$ dominância absoluta $(\%) ; \mathrm{DoR}=$ dominância relativa $(\%) ; \mathrm{FA}=$ frequência absoluta $(\%)$ e $\mathrm{FR}=$ frequência relativa $(\%)$

\begin{tabular}{|c|c|c|c|c|c|c|c|c|c|}
\hline Famílias & $\mathbf{N}^{\circ} \mathbf{S p}$ & $\%$ Sp. & DA & DR & DoA & DoR & FA & FR & IVI \\
\hline Vochysiaceae & 2 & 4,08 & 420,0 & 24,80 & 4,66 & 29,71 & 100,00 & 6,06 & 20,19 \\
\hline Fabaceae & 9 & 18,37 & 193,3 & 11,42 & 2,50 & 15,92 & 100,00 & 6,06 & 11,13 \\
\hline Malpighiaceae & 4 & 8,16 & 136,7 & 8,07 & 1,40 & 8,94 & 100,00 & 6,06 & 7,69 \\
\hline Sapotaceae & 1 & 2,04 & 163,3 & 9,65 & 0,86 & 5,50 & 100,00 & 6,06 & 7,07 \\
\hline Grupo das Mortas & 1 & 2,04 & 93,3 & 5,51 & 0,97 & 6,19 & 100,00 & 6,06 & 5,92 \\
\hline Melastomataceae & 3 & 6,12 & 83,3 & 4,92 & 0,73 & 4,64 & 100,00 & 6,06 & 5,21 \\
\hline Dilleniaceae & 1 & 2,04 & 83,3 & 4,92 & 0,70 & 4,49 & 100,00 & 6,06 & 5,16 \\
\hline Myrtaceae & 2 & 4,08 & 83,3 & 4,92 & 0,50 & 3,17 & 100,00 & 6,06 & 4,72 \\
\hline Ocnaceae & 1 & 2,04 & 76,7 & 4,53 & 0,26 & 1,68 & 100,00 & 6,06 & 4,09 \\
\hline Caryocaraceae & 1 & 2,04 & 43,3 & 2,56 & 0,53 & 3,35 & 100,00 & 6,06 & 3,99 \\
\hline Apocynaceae & 3 & 6,12 & 50,0 & 2,95 & 0,40 & 2,55 & 83,33 & 5,05 & 3,52 \\
\hline Sapindaceae & 1 & 2,04 & 40,0 & 2,36 & 0,43 & 2,73 & 50,00 & 3,03 & 2,71 \\
\hline Annonaceae & 2 & 4,08 & 36,7 & 2,17 & 0,13 & 0,84 & 83,33 & 5,05 & 2,69 \\
\hline Burseraceae & 1 & 2,04 & 40,0 & 2,36 & 0,33 & 2,13 & 33,33 & 2,02 & 2,17 \\
\hline Anacardiaceae & 2 & 4,08 & 13,3 & 0,79 & 0,21 & 1,36 & 66,67 & 4,04 & 2,06 \\
\hline Loganiaceae & 2 & 4,08 & 26,7 & 1,57 & 0,09 & 0,56 & 50,00 & 3,03 & 1,72 \\
\hline Proteaceae & 1 & 2,04 & 26,7 & 1,57 & 0,19 & 1,24 & 33,33 & 2,02 & 1,61 \\
\hline Bignoniaceae & 2 & 4,08 & 20,0 & 1,18 & 0,08 & 0,52 & 50,00 & 3,03 & 1,58 \\
\hline Malvaceae & 1 & 2,04 & 13,3 & 0,79 & 0,04 & 0,23 & 50,00 & 3,03 & 1,35 \\
\hline Nyctaginaceae & 1 & 2,04 & 6,7 & 0,39 & 0,21 & 1,35 & 16,67 & 1,01 & 0,92 \\
\hline Moraceae & 2 & 4,08 & 10,0 & 0,59 & 0,02 & 0,13 & 33,33 & 2,02 & 0,91 \\
\hline Convolvulaceae & 1 & 2,04 & 3,3 & 0,20 & 0,20 & 1,30 & 16,67 & 1,01 & 0,83 \\
\hline Bixaceae & 1 & 2,04 & 6,7 & 0,39 & 0,17 & 1,06 & 16,67 & 1,01 & 0,82 \\
\hline Clusiaceae & 1 & 2,04 & 10,0 & 0,59 & 0,03 & 0,17 & 16,67 & 1,01 & 0,59 \\
\hline Celastraceae & 1 & 2,04 & 6,7 & 0,39 & 0,02 & 0,11 & 16,67 & 1,01 & 0,50 \\
\hline Rubiaceae & 1 & 2,04 & 3,3 & 0,20 & 0,02 & 0,11 & 16,67 & 1,01 & 0,44 \\
\hline Ebenaceae & 1 & 2,04 & 3,3 & 0,20 & 0,01 & 0,04 & 16,67 & 1,01 & 0,41 \\
\hline Total & 49 & 100,00 & 1693,2 & 100,00 & 15,96 & 100,00 & 1650,01 & 100,00 & 100,00 \\
\hline
\end{tabular}

O sucesso da família Vochysiaceae no Cerrado pode estar associado à capacidade de algumas espécies da família em acumular alumínio, se tornando mais competitivas nos solos do Cerrado que, em sua maioria, são solos distróficos com baixa saturação de bases trocáveis, baixa capacidade de troca catiônica, alta acidez e altas concentrações de alumínio no perfil (HARIDASAN, 2000; OLIVEIRA, 2008).
O destaque da família Fabaceae em riqueza é atribuído principalmente a capacidade de fixação de nitrogênio de algumas de suas espécies, além disso, a região central do Brasil é considerada o principal centro de diversificação da família (POLHILL et al., 1981; CAMPELLO, 1998, PEREIRA; SILVA, 2011).

Excluindo o grupo de árvores mortas, as famílias Vochysiaceae (496,70 ind. ha $\left.{ }^{-1}\right)$, Fabaceae (193,30 ind. ha $\left.{ }^{-1}\right)$, 
Sapotaceae (163,30 ind. ha $\left.{ }^{-1}\right)$, Malpighiaceae (136,70 ind. ha $\left.{ }^{1}\right)$ e Melastomataceae, Dilleniaceae e Myrtaceae (83,30 ind. $\mathrm{ha}^{-1}$ ) representaram apenas $26,92 \%$ do total de famílias amostradas, mas detendo $72,70 \%$ da densidade absoluta total.

No que se refere à dominância absoluta, as famílias que mais se destacaram foram: Vochysiaceae $\left(4,92 \mathrm{~m}^{2} \mathrm{ha}^{-1}\right)$, Fabaceae $\left(2,50 \mathrm{~m}^{2} \mathrm{ha}^{-1}\right)$ e Malpighiaceae $\left(1,40 \mathrm{~m}^{2} \mathrm{ha}^{-1}\right)$. Com isso, se tem $11,53 \%$ das famílias amostradas englobando $58,15 \%$ da dominância absoluta total.

As famílias com $100 \%$ de frequência em todas as parcelas amostradas são: Vochysiaceae, Fabaceae, Malpighiaceae, Sapotaceae, Melastomataceae, Dilleniaceae, Myrtaceae e Caryocaraceae. Já as famílias Apocynaceae e Annonaceae estão presentes em cinco das seis parcelas instaladas perfazendo $83,3 \%$ da frequência absoluta. Em uma fitofisionomia como o Cerrado sensu stricto é comum que algumas famílias se sobreponham as demais e, geralmente, as que se sobrepõe são aquelas com maior frequência absoluta, que significa serem mais uniformemente distribuídas (FINGER; FINGER, 2015).

Nas famílias Vochysiaceae, Fabaceae, Malpighiaceae, Sapotaceae, Melastomataceae, Dilleniaceae, Myrtaceae,
Ocnaceae, Caryocaraceae e Apocynaceae, pode-se observar os maiores IVI, onde $37,04 \%$ das famílias amostradas somam $72,77 \%$ do IVI total. As famílias referidas como principais em IVI neste estudo também foram apontadas por Fina e Monteiro (2013) que observaram em um fragmento de Cerrado sensu stricto no Mato Grasso do Sul as famílias Vochysiaceae, Fabaceae e Myrtaceae com os maiores IVI. Rêgo et al. (2015) analisando um fragmento de Cerrado sensu strico em Paranã - TO confirmaram que as famílias Vochysiaceae, Fabaceae e Malpighiaceae são significativas nas áreas de cerrado do Estado do Tocantins.

$\mathrm{Na}$ distribuição do número de indivíduos de cada espécie nas suas respectivas classes diamétricas, observou-se maior concentração dos indivíduos nas três primeiras classes, perfazendo cerca de $88 \%$ dos indivíduos amostrados. Nota-se que a distribuição está dentro do que se espera para florestas nativas em equilíbrio, as quais, caracterizam-se por apresentar distribuição diamétrica decrescente, em forma de 'J-invertido', ou seja, maior quantidade de indivíduos nas classes de tamanhos menores, sendo que isso vai diminuindo com o aumento das classes (Figura 1).

Figura 1. Distribuição diamétrica dos indivíduos amostrados em área de Cerrado sensu stricto, município de Formoso do Araguaia (TO).

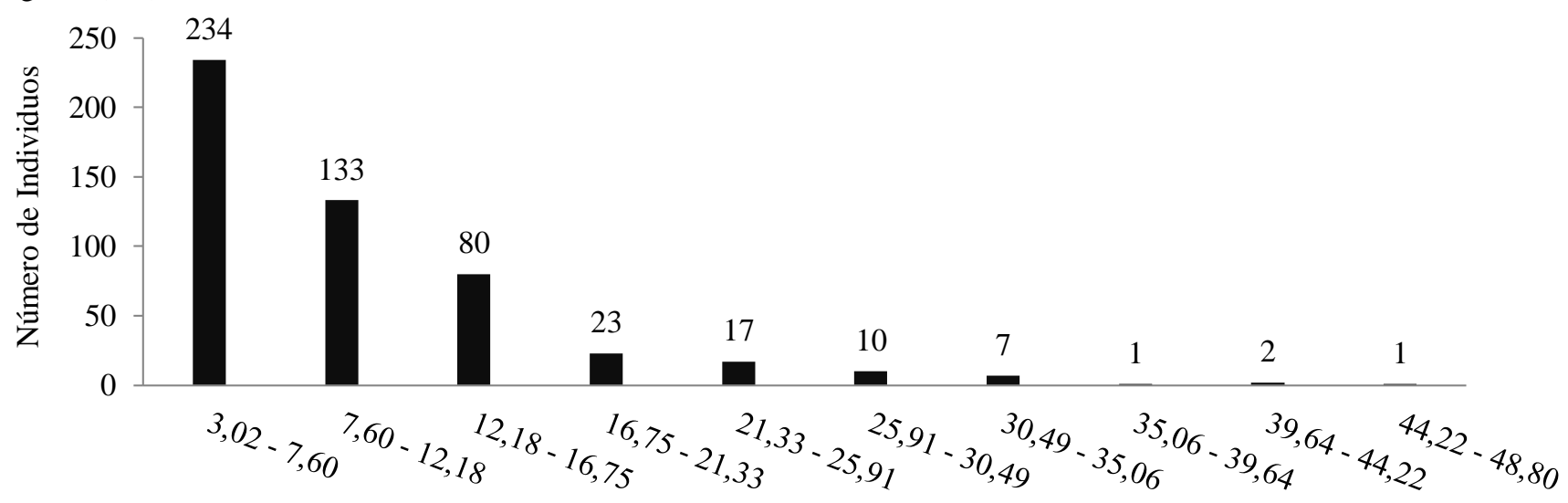

Classe Diamétrica $(\mathrm{cm})$

Conforme se observa na Figura 1, é notória a redução do número de indivíduos a medida que aumenta as classes diamétricas, apresentando característica padrão do "Jinvertido" o que demonstra que a comunidade possui representantes em todas as classes diamétricas e com maior densidade nas classes menores. Pode inferir-se, então, que a área amostrada representa uma população vegetal estável e auto regenerativa, com um balanço positivo entre o recrutamento e a mortalidade dos indivíduos (CARVALHO; MARQUES-ALVES, 2008).

$\mathrm{O}$ padrão de distribuição dos indivíduos em classes de diâmetro, observado neste estudo, também foi verificado por Gomes et al. (2011), Mews et al. (2011), Ferreira et al. (2015), Finger e Finger (2015), Silva et al. (2016) e Silva e Souza (2016) em outras áreas de Cerrado sensu stricto. A distribuição das classes diamétricas sugere o bom estado de conservação da fisionomia pesquisada.

\section{CONCLUSÕES}

O fragmento de Cerrado sensu stricto da Reserva Legal, inserido na Fazenda Bom Sossego tem alta diversidade e bom estado de conservação da estrutura com os valores do índice de diversidade de Shannon-Wienner e equabilidade dentro dos padrões de Cerrado.

As espécies que apresentaram os maiores valores de densidade, dominância e frequência foram Qualea parviflora Mart., Qualea grandiflora Mart., Pouteria ramiflora (Mart.) Radlk. e Byrsonima coccolobifolia Kunth.

As famílias com maiores valores foram Vochysiaceae, Fabaceae, Malpighiaceae e Sapotaceae.

\section{REFERÊNCIAS}

ALVES JÚNIOR, F. T.; LINS C. F.; BRANDÃO, S.; ROCHA, K. D.; SILVA, J. T; MARAGON, L. C. Estrutura diamétrica e hipsométrica do componente arbóreo de um fragmento de mata atlântica, Recife-PE. Revista Cerne, Lavras, v.13, n.1, p.83-95, 2007. 
APG III. An update of the Angiosperm Phylogeny Group classifcation for the orders and families of flowering plants: APG III. Botanical Journal of the Linnean Society, Londres, v.161, p.105-121, 2009.

BRITO, A.; FERREIRA, M. Z.; MELLO, J. M.; SCOLFORO, J. R. S.; OLIVEIRA, A. D.; ACEWRBI, F. W. Comparação entre os métodos de quadrantes e PRODAN para análises florística, fitossociológica e volumétrica. Revista Cerne, Lavras, v.13, n.4, p.399-405, 2007.

CAMPELLO, E. F. C. Sucessão vegetal na recuperação de áreas degradadas. In: DIAS, L. E; MELLO, L. W. V. (Eds.). Recuperação de áreas degradadas. Viçosa: SOBRADE, 1998. p.183-196.

CARVALHO, A. R.; MARQUES-ALVES, S. Diversidade e índice sucessional de uma vegetação de Cerrado sensu stricto na Universidade Estadual de Goiás - UEG, Campus de Anápolis. Revista Árvore, Viçosa, v.32, n.1, p.81-90, 2008.

CARVALHO, M. A. F.; BITTAR, P. A.; SOUZA, P. B.; FERREIRA, R. Q. de S. Florística, fitossociologia e estrutura diamétrica de um remanescente florestal no município de Gurupi, Tocantins. Revista Verde de Agroecologia e Desenvolvimento Sustentável, Pombal, v.11, n.4, p.59-66, 2016.

CERQUEIRA, C. L.; LISBOA, G. S.; STEPKA, T. F.; FRANÇA, L. C. J.; FONSECA, N. C.; ABREU, Y. K. L.; SANTOS, J. C. Florística, Fitossociologia e Distribuição Diamétrica em um Remanescente de Cerrado sensu stricto, Brasil. Revista Espacios, Caracas, v.38, n.23, p.13-29, 2017.

COUTINHO, L. M. O conceito de bioma. Acta Botanica Brasilica, Belo Horizonte, v.20, n.1, p.1-11, 2006.

FELFILI, M. C. Proposição de critérios florísticos, estruturais e de produção para o manejo de cerrado sensu stricto do Brasil central. 2008. 147f. Tese (Doutorado em Ciências Florestais) - Universidade de Brasília, Brasília. 2008.

FELFILI, J. M.; NOGUEIRA, P. E.; SILVA JÚNIOR, M. C.da; MARIMON, B. S.; DELITTI, W. B. C. Composição florística e fitossociológica de um cerrado Sensu Stricto em Água Boa - MT. Acta Botanica Brasilica, Belo Horizonte, v.16, n.1, p.103-112, 2002.

FERREIRA, R. Q. S.; CAMARGO, M. O.; SOUZA, P. B.; ANDRADE, V. C. L. de. Fitossociologia e estrutura diamétrica de um cerrado sensu stricto, Gurupi - TO. Revista Verde de Agroecologia e Desenvolvimento Sustentável, Pombal, v.10, n.1, p.229-235, 2015.

FINA, B. G.; MONTEIRO, R. Análise da estrutura arbustivoarbórea de uma área de cerrado sensu stricto, município de Aquidauana - Moto Grosso do Sul. Revista Árvore, Viçosa, v.37, n.4, p.577-585, 2013.

FINGER, Z.; FINGER, F. A. Fitossociologia em comunidades arbóreas remanescentes de cerrado sensu stricto no Brasil Central. Floresta, Curitiba, v.45, n.4, p.769-780, 2015.

GIÁCOMO, R. G.; CARVALHO, D. C.; PEREIRA, M. G.; SOUZA, A. B.; GAUI, T. D. Florística e fitossociologia em área de campo sujo e cerrado sensu stricto na estação ecológica de Pirapitinga - MG. Ciência Florestal, Santa Maria, v.23, n.1, p.29-49, 2013.

GOMES, L.; LENZA, E.; MARACAHIPES, L.; MARIMON, B. S.; OLIVEIRA, E. A. Comparações florísticas e estruturais entre duas comunidades lenhosas de cerrado típico e cerrado rupestre, Mato Grosso, Brasil. Acta Botanica Brasilica, Belo Horizonte, v.25, n.4, p.865-875, 2011.

HARIDASAN, M. Nutrição mineral de plantas nativas do cerrado. Revista Brasileira de Fisiologia Vegetal, Campinas, v.12, n.1, p.54-64, 2000.

KLINK, C. A.; MACHADO, R. B. A conservação do cerrado brasileiro. Megadiversidade, Rio de Janeiro, v.1, n.1, p.147155, 2005.

LIMA, J. P. C.; LEÃO, J. R. A. Dinâmica de crescimento e distribuição diamétrica de fragmentos de florestas nativa e plantada na Amazônia Sul Ocidental. Floresta e ambiente, Seropédica, v.20, n.1, p.70-79, 2013.

LIMA, R. A. F.; PINHEIRO, I. G.; AGUIRRE, A. G.; CALLARI, C. P. Guia de árvores para o oeste da Bahia. Brasília: IICA, 2013. 206p.

MARTINS, F. R. Estrutura de uma floresta mesófila. São Paulo, UNICAMP, 1991. 246p.

MACHADO, E. L. M.; GONZAGA, A. P. D.; CARVALHO, W. A. C.; SOUZA, J. S.; HIGUCHI, P.; SANTOS, R. M.; SILVA, A. C.; OLIVEIRA-FILHO, A. T. Flutuações temporais nos padrões de distribuição diamétrica da comunidade arbóreo-arbustivo e de 15 populações em um fragmento florestal. Revista Árvore, Viçosa, v. 34, n. 4, p. 723-732, 2010

MEDEIROS, J. D. Guia de campo: vegetação do Cerrado 500 espécies. Brasília, MMA/SBF, 2011. 532p.

MEDEIROS, M. B.; WALTER, B. M. T. Composição e estrutura de comunidades arbóreas de cerrado stricto sensu no norte do Tocantins e sul do Maranhão. Revista Árvore, Viçosa, v.36, n.4, p.679-683, 2012.

MEYER, A. H.; RICKNAGEL, A. B.; STEVENSON, D. D.; BARTOO, R. A. Forest management. New York: The Ronald Press Company; 1961. 282p.

MEWS, H. A.; MARIMON, B. S.; MARACAHIPES, L. FRANCZAK, D. D.; MARIMON-JUNIOR, B. H. Dinâmica da comunidade lenhosa de um cerrado típico na região Nordeste do Estado de Mato Grosso, Brasil. Biota Neotropica, Campinas, v.11, n.1, p.73-82, 2011. 
MMA. Mapeamento do uso e cobertura do cerrado: Projeto TerraClass Cerrado 2013-2016. Brasília: Ministério do Meio Ambiente, 67p., 2015.

MONTEIRO FILHO, P. A.; ROCHA, R. C. Levantamento florístico e fitossociológico na área de proteção ambiental (APA) Encantando em Baliza/GO. Revista OKARA: Geografia em debate, João Pessoa, v.10, n.1, p.45-58, 2016.

OLIVEIRA, P. E. A. M. Fenologia e Biologia Reprodutiva das Espécies de Cerrado. In: S. M. SANO; S. P. ALMEIDA; J. F. RIBEIRO (eds.). Cerrado: ecologia e flora. Planaltina: EMBRAPA -CPAC, 2008. p.273-290.

PAULA, A.; SILVA, A. F.; MARCO JÚNIOR, P.; SANTOS, F. A. M.; SOUZA, A. L. Sucessão ecológica da vegetação arbórea em uma floresta estacional semidecidual, Viçosa, MG, Brasil. Acta Botanica Brasilica, Belo Horizonte, n.18, v.3, p.407-423, 2004.

PEREIRA, B. A. S.; SILVA, M. A. Flora fanerogâmica da Reserva Ecológica do IBGE. In: RIBEIRO, M. L. (ed.). Reserva Ecológica do IBGE: biodiversidade terrestre. Tomo II. IBGE: Rio de Janeiro, 2011. p. 23-37.

POLHILL, R. M.; RAVEN, P. H.; STIRTON, C. H. Evolution and systematics of the Leguminosae. In: POLHILL, R. M.; RAVEN, P. H. (eds.). Advances in legume systematics. Royal Botanic Gardens Kew: Londres, 1981. p.1-26.

RATTER, J. A.; BRIDGEWATER, S.; RIBEIRO, J. F. Analysis of the floristic composition of the Brazilian Cerrado vegetation III: Comparison of the woody vegetation of 376 areas. Edinburgh Journal of Botany, Cambridge, v.60, n.1, p.57-109, 2003.

RÊGO, A. B. M. L.; SOUZA, P. B.; SILVA, R. R.; RÊGO, P. L. Composição florística e estrutural de um componente arbóreo em área de cerrado, Paranã - TO, Revista Verde de Agroecologia e Desenvolvimento Sustentável, Pombal, v.10, n.5, p.108-114, 2015.
RIBEIRO, J. F. WALTER, B. M. T. Fitofisionomias do bioma Cerrado. In: SANO, S. M; ALMEIDA, S. P. RIBEIRO, J. F. Cerrado: ambiente e flora. Brasília: Embrapa Cerrados, 2008. p 89-166.

SANTOS, L. J. dos; MARMONTEL, C. V. F.; MARTINS, T. M.; MELO, A. G. C. de. Fitossociologia de cerrado Sensu stricto localizado no município de Carbonita - MG. Revista Cientifica Eletrônica de Engenharia Florestal, Garça, v.15, n.1, p.77-90, 2010.

SEPLAN. Atlas do Tocantins: subsídios ao planejamento da gestão territorial. $6^{a}$ ed., Palmas: Secretaria do Planejamento e da Modernização da Gestão Pública, 80 p., 2012.

SHEPERD, G. J. FITOPAC 2: manual do usuário. Campinas: UNICAMP, 2010. 91p.

SILVA, G. O.; SOUZA, P. B. Fitossociologia e estrutura diamétrica de um fragmento de cerrado sensu stricto, Gurupi - TO. Revista Desafios, Palmas, v.3, n. Especial, p.22-29, 2016.

SILVA, R. A.; PAIXÃO, E. C.; CUNHA, C. N.; FINGER, Z. Fitossociologia da comunidade arbórea de cerrado sensu stricto do Parque Nacional da Chapada dos Guimarães. Nativa, Guarantã do Norte, v.4, n.2, p.82-86, 2016.

SILVA NETO, V. L.; OLIVEIRA, A. L.; FERREIRA, R. Q. S.; SOUZA, P. B.; VIOLA, M. R. Fitossociologia e distribuição diamétrica de uma área de cerrado sensu stricto, Dueré-TO. Revista de Ciências Ambientais, Canoas, v.10, n.1, p.91-106, 2016.

SILVA NETO, V. L.; OLIVEIRA, A. L.; SANTOS, A. F.; LOURENÇO, S. T. S. Distribuição diamétrica e estrutura fitossociológica de cerrado sensu stricto em Gurupi - TO. Enciclopédia Biosfera, Goiânia, v.13, n.23, p.495-508, 2016.

SIMINSKI, A.; MANTOVANI, M.; REIS, M. S.; FANTINI, A. C. Sucessão florestal secundária no município de São Pedro de Alcântara, litoral de Santa Catarina: Estrutura e diversidade. Ciência Florestal, Santa Maria, v.14, n.1, p.2133, 2004. 